\title{
Perspectives on immunotherapy via oncolytic viruses
}

\author{
Alberto Reale, Adriana Vitiello, Valeria Conciatori, Cristina Parolin, Arianna Calistri and Giorgio Palü
}

\begin{abstract}
Background: With few exceptions, current chemotherapy and radiotherapy protocols only obtain a slightly prolonged survival with severe adverse effects in patients with advanced solid tumors. In particular, most solid malignancies not amenable to radical surgery still carry a dismal prognosis, which unfortunately is also the case for relapsing disease after surgery. Even though targeted therapies obtained good results, clinical experience showed that tumors eventually develop resistance. On the other hand, earlier attempts of cancer immunotherapy failed to show consistent efficacy. More recently, a deeper knowledge of immunosuppression in the tumor microenvironment (TME) allowed the development of effective drugs: in particular, monoclonal antibodies targeting the so-called immune checkpoint molecules yielded striking and lasting effects in some tumors. Unfortunately, these monoclonal antibodies are not effective in a majority of patients and are ineffective in several solid malignancies. Furthermore, due to their mechanism of action, checkpoint inhibitors often elicit autoimmune-like disease.

Main body: The use of viruses as oncolytic agents (OVs) was considered in the past, while only recently OVs revealed a connection with immunotherapy. However, their antitumoral potential has remained largely unexplored, due to safety concerns and some limitations in the techniques to manipulate viruses. OV research was recently revived by a better knowledge of viral/cancer biology and advances in the methodologies to delete virulence/immune-escape related genes from even complex viral genomes or "to arm" OVs with appropriate transgenes. Recently, the first oncolytic virus, the HSV-1 based Talimogene Laherparepvec (T-VEC), was approved for the treatment of non-resectable melanoma in USA and Europe.

Conclusion: OVs have the potential to become powerful agents of cancer immune and gene therapy. Indeed, in addition to their selective killing activity, they can act as versatile gene expression platforms for the delivery of therapeutic genes. This is particularly true for viruses with a large DNA genome, that can be manipulated to address the multiple immunosuppressive features of the TME. This review will focus on the open issues, on the most promising lines of research in the OV field and, more in general, on how OVs could be improved to achieve real clinical breakthroughs in cancers that are usually difficult to treat by immunotherapy.
\end{abstract}

Keywords: Oncolytic virus, Oncolytic virotherapy, Cancer immunotherapy, Cancer gene therapy, Oncolytic HSV-1, Tumor microenvironment

\section{Background}

The pharmacological therapy of cancer represents one of the greatest challenges for contemporary medicine. State-of-the-art chemotherapy and radiotherapy protocols can be curative in some hematologic malignancies, such as Hodgkin lymphoma and acute lymphoid leukemia (ALL), and can be successfully combined with other therapeutic solutions like autologous stem cell transplantation

* Correspondence: giorgio.palu@unipd.it

Department of Molecular Medicine, University of Padua, Via A. Gabelli, 63, 35121 Padua, Italy

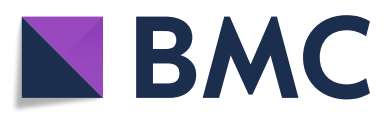

$[1,2]$. Targeted therapies have also emerged that changed the natural course of diseases like chronic myeloid leukemia or promyelocytic myeloid leukemia [3, 4]. Even for ALL resistant to current therapies, the use of chimeric antigen receptor (CAR)-T cellular therapy provided a major breakthrough [5].

The situation is much bleaker for non-hematologic neoplasms. With very few exceptions, in this case, the hope of a cure rests mainly on the possibility of a radical surgical excision at the moment of diagnosis. If this is not possible, due to extensive local invasion or metastatic dissemination,

(c) The Author(s). 2018 Open Access This article is distributed under the terms of the Creative Commons Attribution 4.0 International License (http://creativecommons.org/licenses/by/4.0/), which permits unrestricted use, distribution, and reproduction in any medium, provided you give appropriate credit to the original author(s) and the source, provide a link to the Creative Commons license, and indicate if changes were made. The Creative Commons Public Domain Dedication waiver (http://creativecommons.org/publicdomain/zero/1.0/) applies to the data made available in this article, unless otherwise stated. 
prognosis remains dismal $[6,7]$. Great expectations were associated with targeted therapies, such as small molecule tyrosine kinase inhibitors (TKIs) or monoclonal antibodies directed against receptors overexpressed by cancer cells. Even though these approaches obtained good results in selected patients, in terms of prolonged survival, with a good toxicity profile, it soon became evident that tumors usually develop resistance $[8,9]$.

Another possible therapeutic strategy is immunotherapy. Although it has been known for quite a long time that the immune system can recognize and kill cancer cells, previous attempts of immunotherapy based on the administration of recombinant cytokines, anti-cancer vaccines or in vitro expanded tumor infiltrating lymphocytes (TILs) did not provide enough efficacy [10, 11]. Still, there were some remarkable exceptions, as a small subset of metastatic melanoma and of clear cell renal carcinoma patients showed long-term remissions after treatment with high doses of recombinant interleukin 2 (rIL-2) [12]. In recent years, new light was shed on mechanisms involved in cancer immunology, and, especially, on the immunosuppressive features of the tumor microenvironment (TME), which mediate escape from tumoricidal immune responses. In particular, cancer has the ability to exploit mechanisms involved in the maintenance of immune peripheral tolerance, either i) directly, by expressing immune checkpoint molecule ligands which dampen the activity of cytotoxic $\mathrm{T}$ cells, such as Programmed Death Ligand-1 (PDL-1), or ii) indirectly, by recruiting immune cells with immunosuppressive features, such as CD4+ CD25+ Foxp3+ $\mathrm{T}$ regulatory cells (Tregs), immature myeloid-derived suppressor cells (MDSCs), or M2 macrophages [13, 14]. These cells usually express checkpoint molecule ligands and secrete soluble cytokines (e.g. IL-10) or enzymes (arginase and IDO) that hinder cytotoxic $\mathrm{T}$ responses. These and other actors, like cancer associated fibroblasts and downregulation of MHC class I molecules by cancer cells, are probably playing a role in TME immunosuppression. Based on these considerations, new cancer immunotherapies were developed, based on checkpoint inhibition by means of monoclonal antibodies directed against Cytotoxic T Lymphocyte Antigen 4 (CTLA-4), Programmed Death-1 (PD-1), or its ligands PDL-1 and PDL-2 [15]. Anti-CTLA-4 humanized antibodies, as ipilimumab, were the first to show therapeutic efficacy against melanoma [16]. On the other hand, anti-PD1 and anti-PDL1 mAbs seem to have a broader spectrum of action (including NSCLC and possibly small subsets of pancreatic and breast cancer), while triggering less autoimmune toxicity [17]. However, also in cancer types considered susceptible to checkpoint inhibitors, more than $50 \%$ of patients fail to respond to treatment. In this context, the combination of different checkpoint inhibitors
(anti-CTLA-4 and anti-PD1 Abs) yielded better results in melanoma patients, but with increased toxicity [18].

Oncolytic viruses (OVs) are defined as viruses able to selectively replicate in and kill cancer cells [19]. The history of OVs is quite long, since already at the beginning of the twentieth century physicians observed that cancer patients experienced partial disease remissions after natural infections [20]. It was, therefore, hypothesized that cancer cells were somehow more vulnerable to viral infections, and that attenuated viral strains could be used in cancer therapy. However, many factors, including safety concerns, the development of cytotoxic chemotherapy, and the lack of tools to manipulate viruses, hindered research in this field. In recent years OV studies were revived by better knowledge of viral gene function and advancements in molecular biology, which allow precise modifications of viral genomes to maximize both efficacy and safety. Over the last years a new paradigm emerged according to which OVs might also function as a form of immunotherapy [21]. Indeed, it has been shown that the proinflammatory stimuli provided by viruses can overcome the TME immunosuppression and, thereby, elicit a systemic antitumoral immune response. Such a response was observed also when OVs were injected locally (intratumoral injection), rather than systemically [22]. It was demonstrated that the first OV approved for cancer treatment in North America and Europe, the HSV-1 based talimogene laherparepvec (T-VEC), has an immunological mechanism of action, which also causes the regression of uninjected and uninfected metastases [23].

Nevertheless, OVs are still not powerful enough, especially for scarcely immunogenic or immunosuppressive solid tumors, which unfortunately are quite frequent in the population, like pancreatic adenocarcinoma, triple negative breast cancer, hepatocellular carcinoma [24-26]. This lack of efficacy is somehow unexpected, as OVs should make the TME significantly more immunogenic due to inflammation and the presence of viral antigens. Such a consideration fuels the feeling that major improvements in the OV therapy field are at hand.

This review will focus on open issues regarding OVs, and especially their interaction with the TME and the host immune system. The answer to these questions will probably be crucial to fully exploit the therapeutic potential of OVs.

\section{Main text}

As explained above, OVs are emerging as a new, promising form of immunotherapy. In recent years a remarkable array of different OVs has been tested in preclinical cancer models or in phase I/II clinical trials [27]. This plethora of viruses includes, among the others, attenuated strains of human pathogens, such as adenoviruses (AdVs) [28], 
herpes simplex type 1 (HSV-1) [29], vaccinia virus (VACV) [30], measles [31], mumps virus [32] and influenza A virus [33], or viruses that are naturally poorly pathogenic for humans, including the orthoreovirus strain T3D [34], Newcastle Disease Virus (NDV) [35], vesicular stomatitis virus (VSV) [36], Maraba Virus [37], the rodent $\mathrm{H}-1$ parvovirus [38] and the picornavirus Mengovirus [39], a long list far from being complete [Table 1]. Perhaps the most striking common feature of these heterogeneous OVs is their outstanding safety profile. Indeed, severe adverse effects were very rare and it was unusual that therapy had to be discontinued due to toxicity [40]. Unfortunately, safety was not always matched by efficacy, and so far only the HSV-1 based talimogene laherparepvec was effective enough to be authorized for routine clinical use. Also, efficacy was markedly higher in immunogenic tumors such as melanoma [41].

Therefore, despite the fact that OVs hold great therapeutic potential, it is clear that they need to be further improved. A better understanding of their in vivo mechanisms of action and pharmacokinetics, as well as a clearer picture of the complex interplay between viruses and host are some of the crucial aspects to be further elucidated to design safer and more effective OVs. Under this respect, different questions remain to be addressed:

1. How "attenuated" should an attenuated OV be? The question may sound trivial, the answer being "attenuated enough to replicate only in cancer cells". However, the application of this oversimplified principle can have dire consequences, as it does not consider the real complexity of tumors. Many OVs were designed to be able to replicate only in actively dividing cells, for example by deletion of specific genes (like HSV or VACV thymidine kinase and ribonucleotide reductase), according to the idea that cancer cells are actively replicating while healthy cells are not [42]. Unfortunately, many cancer cells within a tumor mass are not undergoing replication. To make things worse, many non-tumoral cells are present in the TME (including macrophages, endothelial cells, lymphocytes, fibroblasts, MDSCs). It has been shown that these cells do not support the replication of OVs designed according to the aforementioned principle and can, therefore, protect malignant cells from viral diffusion [43]. Furthermore, due to the well known cancer heterogeneity, it is hazardous to assume that all cancer cells in all patients will display the same specific molecular characteristic, like mutations within certain oncosuppressor genes and/or overexpression of single pathways [44] and to assume that OV specificity towards cancer cells could rely only on these features
$[45,46]$. Indeed, evidence demonstrate that the increased susceptibility of cancer cells towards viral replication is the result of variable combinations of alterations mainly in antiviral response and cell cycle regulation pathways. Thus, a more sensible approach for generating OVs, when starting from well-known human pathogens, might be to attenuate them in such a way that they cannot cause the dangerous forms of disease they are associated with (Table 1). In the case of HSV-1, in immunocompetent adults nearly all severe morbidity and mortality is caused by dissemination and replication in neurons, resulting in encephalitis. Therefore, genome modifications that attenuate HSV1 virulence in neurons might be sufficient to generate a safe OV, despite the fact that the virus retains, at least partially, its ability to replicate in "healthy" fibroblasts or epithelial cells. This feature might even be useful, as it enables the virus to be more effective in the TME, as appears to be in the case of T-VEC.

2. What defines a solid in vitro model to screen for selectivity of OVs towards cancer cells? This question persists despite the fact that, according to what we have suggested above, the focus could be shifted from "unable to replicate in nonmalignant cells" to "unable to replicate in specific target cells relevant for human disease" (neurons in the case of HSV). The problem is associated with the definition itself of "nonmalignant" applied to tissue culture cells. Cell lines, even when incapable of forming tumors once inoculated in immunosuppressed mice, are often immortalized and have very different features from their in vivo counterparts, which can lead to OVs replication in these "healthy" cells. Furthermore, cell lines (including cancer cell lines) are often unpredictable in their susceptibility to viral replication: even viruses with broad cell tropism will occasionally produce very low titers in some cell lines [unpublished observations]. This raises the issue of finding a real "healthy" cell line in which the OV under evaluation is not replicating because the cell line does not have malignant characteristics, rather than because that cell line is characterized by refractoriness to that virus. Primary cells, although technically more demanding, could partially overcome some of these difficulties. On the other hand, organoids derived from malignant and healthy tissue, that are becoming a widely employed in vitro model for several types of studies, would have the further advantage of letting cells grow in a $3 \mathrm{D}$ environment, more closely mimicking the in vivo situation [47]. Thus, organoids might represent 


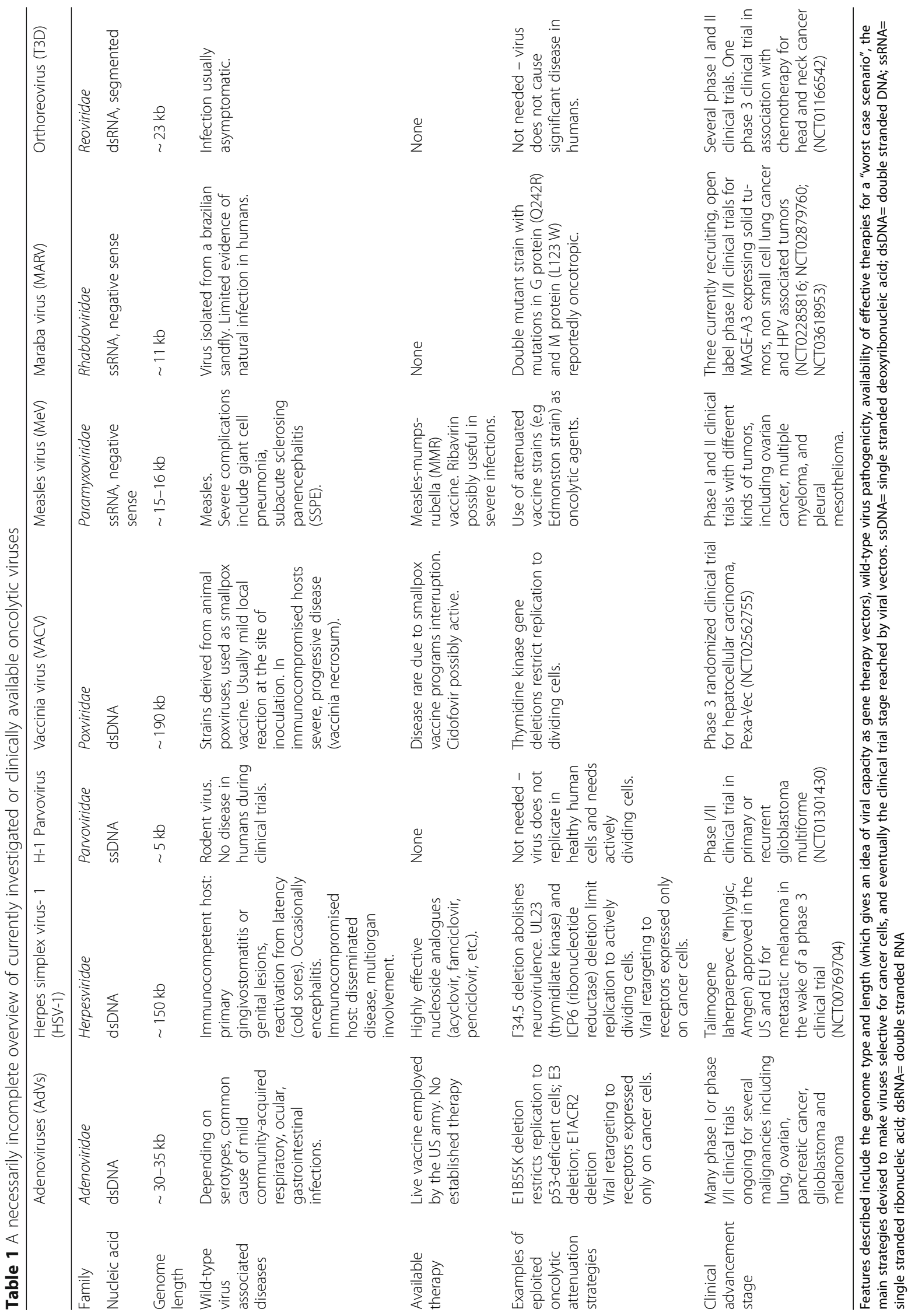


the best in vitro model to test OV specificity towards cancer cells.

3. What is the most appropriate animal model to test safety and efficacy of OVs? Most studies so far relied on SCID or nude mice, which are a readily available model in which murine or human cancer cell lines can thrive because of immunosuppression [48]. Still, this feature (especially the absence of a competent $\mathrm{T}$ cell response) profoundly alters the mechanism of action of OVs. In these models, direct oncolysis by the virus could actually be the main effector mechanism that prolongs survival of the animals, which is probably not what happens in human patients. On the other hand, the use of immunocompetent mice could partially overcome this difficulty. However, the differences between the murine and human immune systems are still a hurdle, especially when the ability of the OV of influencing the immune response against cancer cells is under evaluation. Humanized mice, i.e.completely immunodeficient mice which receive a human hematopoietic stem cells (HSC) transplant plus human fetal thymus and liver tissue to guarantee $\mathrm{T}$ cell maturation, could provide an answer to this difficult problem [49].

4. What is the exact role of the host immune system/ $\mathrm{OV}$ interaction in determining the success of virotherapy for tumors? Some investigators argue that the immune system has a deleterious effect, since it could wipe away the OV, especially if deriving from a human pathogen widely present in the population, before it can kill a sufficient number of cancer cells. As a consequence, efforts were spent on concealing the virus from the immune system, or on using viruses for which a preexisting immunity in the general population is unlikely [50]. However, this concept is clearly rooted in the older paradigm of OVs directly killing cancer cells rather than in the recent idea of OVs as tools for immunotherapy. Indeed, recent data strongly suggest that the release of danger signals and inflammation due to OV replication, along with immune system activation against infected cells, account for an important part of the antitumoral potential of the OV itself [51]. Furthermore, in the case of T-VEC, no differences in therapeutic effects were observed between HSV-1 seronegative and seropositive patients. Recently, a study using a mouse model of melanoma treated with the paramyxovirus NDV showed enhanced antitumoral activity in mice with preexisting immunity to NDV [52]. Finally, after intratumoral treatment with $\mathrm{T}$ VEC, it was observed that uninjected lesions, including some visceral metastases, underwent regression. In a recent clinical trial, regression was heralded by activated CD8+ lymphocytes infiltration and was enhanced by checkpoint inhibitors [53]. Such a pattern is consistent with an immune response elicited by viral injection in multiple accessible lesions but effective also against uninjected lesions in which the virus was not detectable.

5. What is the best route of administration for an OV? It is often stated that an ideal OV should be systemically injectable, for some good reasons: essentially, the possibility to infect both primary tumor and metastases, and the fact that this route is relatively non-invasive and injections can be frequently repeated [54]. However, although some OVs (VACV, T3D orthoreovirus, $\mathrm{H}-1$ parvovirus), were administered intravenously to human patients without severe side effects, the most used route is the local (intratumoral) injection [55]. This is the case also for the only approved OV, T-VEC. Intratumoral delivery is usually chosen because of safety concerns after intravenous injection, or, especially in the case of HSV-1, to minimize the chance that preexisting circulating antibodies might neutralize the virus before it reaches its target, as discussed above [56]. Nevertheless, as mentioned above, in the case of T-VEC, despite the intratumoral injection, uninjected lesions and visceral metastases displayed a regression, likely due to the immune response elicited by the virus [41].

6. Viruses have an important feature, which makes them particularly appealing as cancer therapeutics: they are not just cancer cell killers or a proinflammatory stimulus, but they can also serve as platforms for the delivery/expression of transgenes. This feature allows the development of OVs "armed" with therapeutic genes, some of which are already under evaluation in clinical trials. One example is again T-VEC that, in addition to specific mutations within viral genes, carries two copies of the human granulocyte-monocyte colony stimulating factor (hGM-CSF) encoding sequence, under the transcriptional control of the human CMV immediate early promoter [57]. However, most of these engineered viruses only express a single immunostimulatory cytokine or ligand $[58,59]$. As a result, these OVs do not exploit the wealth of information that was recently accumulated on cancer immunology and the TME [60], and may even be outdated. For instance, recent comprehensive reviews cast a dubious light on the usefulness of hGM-CSF in cancer immunotherapy [61]. The issue with cancer immunotherapy is not simply boosting a "sleeping" immune response, but the fact that cancer cells actively use 
immunosuppressive mechanisms and recruit tolerogenic cells. OVs could be used to locally deliver high and constant concentrations of single-chain antibodies or other protein ligands that disrupt those immunosuppressive features. Under this respect, many different strategies can be devised, including the expression of enzymes that degrade the abundant extracellular matrix present in some tumors (desmoplastic reaction) or of dominant negative forms of immunosuppressive cytokines (for example TGF- $\beta$ ) [62].

7. More specifically, could there be space for engineered OVs expressing checkpoint inhibitors, as single chain antibodies, whole antibodies, or proteins that may have the same function? Potent immune checkpoint inhibitors (CKIs) which are delivered systemically are already available, and most investigators are focusing on synergism between existing checkpoint inhibitors and OVs [63]. However, CKIs expressed as therapeutic genes by OVs would probably have the advantage of a prolonged and localized delivery in the TME, which might, in principle, avoid the autoimmune side effects usually associated with systemic CKIs.

8. Is there the possibility of a more extensive cellspecific reprogramming of viruses? Ideally, once an
OV reaches the TME, it should produce different effects in different types of cells. Of course, it should replicate in cancer cells and cause their death while sparing surrounding normal tissue and/ or non causing severe diseases. However there could be further nuances. For example, an OV could be designed to specifically trigger a Th1 phenotype in infected macrophages or to replicate also in cancer associated fibroblasts or endothelial cells that might become a more persistent "factory" of therapeutic gene products. Transgenes under the transcriptional control of cell-specific promoter might serve to this end. While "promoter retargeting" has been explored to enhance viral replication in cancer cells, such an approach to the diversity of the TME has not been investigated yet, at least to our knowledge.

\section{Conclusions}

Cancer immunotherapy is establishing new paradigms in the treatment of advanced stage solid malignancies. Together with immune checkpoint inhibitors, OVs are increasingly recognized as a promising therapeutic tool in this field. The use of OVs on patients has become a clinical reality in the case of talimogene laherparepvec, also known as T-VEC, for metastatic melanoma, and recent

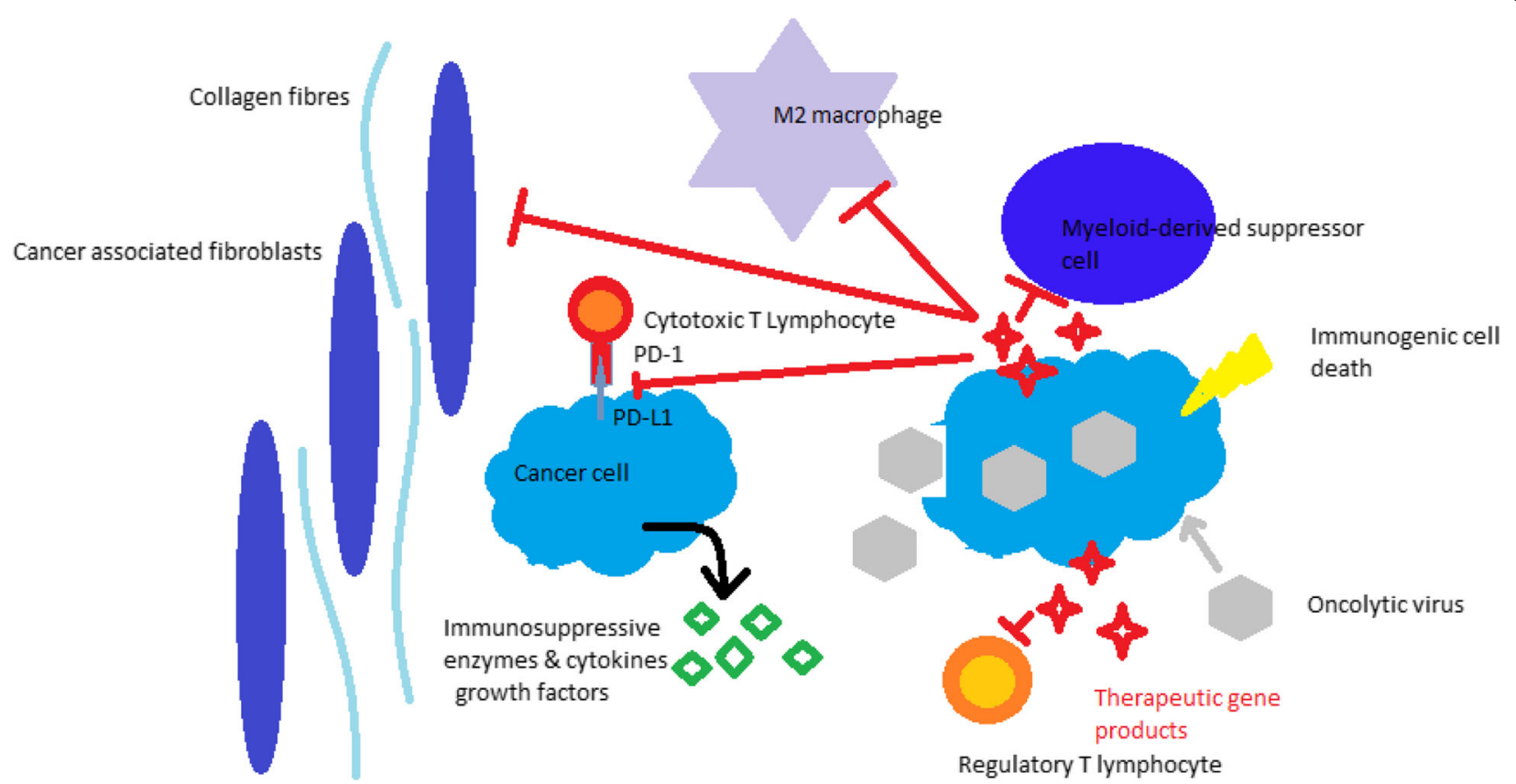

Fig. 1 Schematic representation of cancer cells (cyan) in their immunosuppressive microenvironment, which they shape by secreting cytokines and growth factors. Immune checkpoint molecules and immunosuppressive enzymes released in the milieu inhibit cytotoxic T lymphocytes. Infection of cancer cells by an oncolytic virus (OV, gray) disrupts the immunosuppressive features of the microenvironment by triggering immunogenic cell death and releasing proinflammatory substances. OVs can also be armed with therapeutic genes targeting non malignant cells that support tumor growth and immune escape, such as cancer associated fibroblasts, M2 macrophages, myeloid derived suppressor cells (MDSCs), and regulatory T lymphocytes 
clinical trials strongly suggest that the combination of talimogene with CKIs could be particularly effective in this setting $[23,53]$. Despite this successes, OV treatment of cancers other than melanoma, which is usually considered a very immunogenic tumor, has given limited clinical results $[64,65]$. Several recent studies have been trying to characterize the antitumoral immune response after $\mathrm{OV}$ therapy both in mouse models and in patients enrolled in clinical trials $[53,66]$. However, current basic and translational research on OVs is mainly focused on safety (which, however, has never been a real issue over decades of clinical trials), on various combinations of OVs with chemo and radiotherapy or CKIs, and on the quest for "exotic" non-human viruses, whose ability to infect and lyse a significant number of human cancer cells in vivo remains questionable [67].

The feeling that OVs are not being exploited to their potential is increased by the lack of new ideas on the use of OVs as platforms to express factors aimed at increasing their killing ability and the immunomodulatory effect. Under this respect, there have not been many novelties in the last years, at least to our knowledge. Indeed, in the field of OVs, viral engineering has been mainly employed for the attenuation of the human pathogen under evaluation, for its transcriptional [68] or receptorial [69] retargeting, for the expression of suicide genes or single immunostimulatory cytokines (as in the case of talimogene). Thus, there is wide space for the design of innovative OVs to better achieve, for instance, TME modulation. The ideal candidate would be a large dsDNA virus that can allow the insertion of multiple transgenes within its genome, without losing its ability to replicate in and kill cancer cells, and for which "robust" gene editing techniques are available.

Finally, the big challenge that OVs are facing is the therapy of immunologically "cold" tumors which are usually failing to respond to checkpoint inhibitors due to the absence of a lymphocyte infiltrate. The presence of a virus (especially of a replication competent virus) can profoundly alter the TME by enhancing the immune cell infiltrate and generating proinflammatory cues. Is this enough to make cold tumors sensitive to immune checkpoint inhibition? It must be considered that these tumors often display an immunosuppressive immune cell infiltrate or a fibrotic microenvironment, which could protect malignant cells from the immunogenic stimuli provided by the virus. Armed OVs might be once again the solution to this problem. Indeed, therapeutic gene products, released at high concentrations in situ by infected cells, could synergize with OVs by killing immunosuppressive cells or inhibiting their activity. Furthermore, OVs could be engineered to express enzymes that degrade the fibrotic extracellular matrix, thus helping to tackle very "difficult" tumor microenvironments [Fig. 1].

\section{Abbreviations}

CKI: Checkpoint Inhibitors; CTLA-4: Cytotoxic T Lymphocyte Antigen 4; hGMCSF: human Granulocyte Monocyte Colony Stimulating Factor; HSV: Herpes Simplex Virus; OV: Oncolytic Virus; PD-1: Programmed Death 1; PD-

L1: Programmed Death Ligand 1; TME: Tumor Microenvironment

\section{Availability of data and materials}

Not applicable.

\section{Authors' contributions}

All Authors contributed in writing the review. All authors read and provided critical revision of the manuscript and approved the final version.

Ethics approval and consent to participate

Not applicable.

\section{Consent for publication}

Not applicable.

\section{Competing interests}

The Authors declare that they have no competing interests.

\section{Publisher's Note}

Springer Nature remains neutral with regard to jurisdictional claims in published maps and institutional affiliations.

Received: 18 October 2018 Accepted: 20 December 2018

Published online: 11 February 2019

\section{References}

1. Ansell SM. Hodgkin lymphoma: diagnosis and treatment. In: Mayo Clinic proceedings (2015). (Vol. 90, no. 11, pp. 1574-1583). Elsevier.

2. Hefazi M, Litzow MR. Recent advances in the biology and treatment of $T$ cell acute lymphoblastic leukemia. Curr Hematol Malig Rep. 2018:1-10.

3. Soverini S, Mancini M, Bavaro L, Cavo M, Martinelli G. Chronic myeloid leukemia: the paradigm of targeting oncogenic tyrosine kinase signaling and counteracting resistance for successful cancer therapy. Mol Cancer. 17(1).

4. Kayser S, Schlenk RF, Platzbecker U. Management of patients with acute promyelocytic leukemia. Leukemia. 2018;1.

5. Phelan KW, Advani AS. Novel Therapies in Acute Lymphoblastic Leukemia. Current hematologic malignancy reports.2018; 1-11.

6. Siegel RL, Miller KD, Jemal A. Cancer statistics, 2018. CA Cancer J Clin. 2017;68.

7. de Groot PM, Wu CC, Carter BW, Munden RF. The epidemiology of lung cancer. Transl Lung Cancer Res. 2018;7(3).

8. Gainor JF, Shaw AT. Emerging paradigms in the development of resistance to tyrosine kinase inhibitors in lung cancer. J Clin Oncol. 2013;31.

9. Jarboe J, Gupta A, Saif W. Therapeutic human monoclonal antibodies against Cancer. 2014.In: Steinitz M. (eds) human monoclonal antibodies. Methods in molecular biology, vol 1060. Humana press, Totowa, NJ.

10. Hinrichs CS, Rosenberg SA. Exploiting the curative potential of adoptive Tcell therapy for cancer. Immunol Rev. 2014;257(1).

11. Palucka K, Banchereau J. Dendritic-cell-based therapeutic cancer vaccines. Immunity. 2013(1):39.

12. Rosenberg SA. IL-2: the first effective immunotherapy for human cancer. The Journal of Immunology. 2014;192(12).

13. Gajewski TF, Schreiber H, Fu YX. Innate and adaptive immune cells in the tumor microenvironment. Nature immunology. 2013;14(10).

14. Liu Y, Cao X. Immunosuppressive cells in tumor immune escape and metastasis. J Mol Med. 2016;94(5).

15. Seidel JA, Otsuka A, Kabashima K. Anti-PD-1 and anti-CTLA-4 therapies in Cancer: mechanisms of action, efficacy, and limitations. Front Oncol. 2018;8.

16. Hodi FS, O'day SJ, McDermott DF, Weber RW, Sosman JA, Haanen J., et al. Improved survival with ipilimumab in patients with metastatic melanoma. New England J Med. 2010; 363(8).

17. Palmieri DJ, Carlino MS. Immune Checkpoint Inhibitor Toxicity. Curr Oncol Rep. 2018;20:9.

18. Larkin J, Chiarion-Sileni V, Gonzalez R, Grob JJ, Cowey CL, Lao CD, et al. Combined Nivolumab and Ipilimumab or Monotherapy in Previously Untreated Melanoma. N Engl J Hum Serv. 2015;373(1). 
19. Russell SJ, Peng KW, Bell JC. Oncolytic virotherapy. Nature Biotechnology. 2012;30(7).

20. Kelly E, Russell SJ. History of oncolytic viruses: genesis to genetic engineering. Mol Ther. 2007;15(4).

21. Kaufman HL, Kohlhapp FJ, Zloza A. Oncolytic viruses: a new class of immunotherapy drugs. Nature reviews Drug discovery. 2015;14(9).

22. Nguyen T, Avci NG, Shin DH, Martinez-Velez N, Jiang H. Tune Up In Situ Autovaccination against Solid Tumors with Oncolytic Viruses. Cancers. 2018; 10(6).

23. Puzanov I, Milhem MM, Minor D, Hamid O, Li A, Chen L, et al Talimogene laherparepvec in combination with ipilimumab in previously untreated, unresectable stage IIIB-IV melanoma. J Clin Oncology. 2016;34(22).

24. Noonan AM, Farren MR, Geyer SM, Huang Y, Tahiri S, Ahn D, et al. Randomized phase 2 trial of the oncolytic virus pelareorep (reolysin) in upfront treatment of metastatic pancreatic adenocarcinoma. Molecular Therapy. 2016;(6):24.

25. Suryawanshi YR, Zhang T, Essani K. Oncolytic viruses: emerging options for the treatment of breast cancer. Med Oncology. 2017;34(3).

26. Jebar AH, Errington-Mais F, Vile RG, Selby PJ, Melcher AA, Griffin S. Progress in clinical oncolytic virus-based therapy for hepatocellular carcinoma. J Gen Virol. 2015;96(7)

27. Pol J, Buqué A, Aranda F, Bloy N, Cremer I, Eggermont A, et al. Trial Watch-Oncolytic viruses and cancer therapy. Oncoimmunology. 2016;5(2).

28. Shaw AR, Suzuki M. Recent advances in oncolytic adenovirus therapies for cancer. Current opinion in virology. 2016;21.

29. Sokolowski NA, Rizos H, Diefenbach RJ. Oncolytic virotherapy using herpes simplex virus: how far have we come? Oncolytic virotherapy. 2015;4.

30. Haddad D. Genetically engineered vaccinia viruses as agents for cancer treatment, imaging, and transgene delivery. Front Oncol. 2017;7.

31. Aref S, Bailey K, Fielding A. Measles to the rescue: A review of oncolytic measles virus. Viruses. 2016;8(10)

32. Ammayappan A, Russell SJ, Federspiel MJ. Recombinant mumps virus as a cancer therapeutic agent. Molecular Therapy-Oncolytics. 2016;3.

33. Pizzuto MS, Silic-Benussi M, Ciminale V, Elderfield RA, Capua I, Barclay WS. An engineered avian-origin influenza $A$ virus for pancreatic ductal adenocarcinoma virotherapy. Journal of General Virology. 2016;97(9).

34. Bourhill T, Mori Y, Rancourt DE, Shmulevitz M, Johnston RN. Going (Reo) viral: factors promoting successful revival oncolytics infection. Viruses. 2018;10(8)

35. Tayeb S, Zakay-Rones Z, Panet A. Therapeutic potential of oncolytic Newcastle disease virus: a critical review. Oncolytic virotherapy. 2015;4.

36. Bishnoi S, Tiwari R, Gupta S, Byrareddy SN, Nayak D. Oncotargeting by Vesicular Stomatitis Virus (VSV): Advances in Cancer Therapy. Viruses. 2018 ; 10(2).

37. Pol JG, Zhang L, Bridle BW, Stephenson KB, Rességuier J, Hanson S, et al. Maraba virus as a potent oncolytic vaccine vector. Molecular Therapy. 2014; 22(2)

38. Angelova AL, Witzens-Harig M, Galabov AS, Rommelaere J. The Oncolytic Virotherapy era in cancer management: prospects of applying $\mathrm{H}-1$ parvovirus to treat blood and solid cancers. Frontiers in oncology.2017; 7.

39. Ruiz AJ, Hadac EM, Nace RA, Russell SJ. MicroRNA-detargeted mengovirus for oncolytic virotherapy. J Virol. 2016; JVI-02810.

40. Matsuda T, Karube H, Aruga A. A Comparative Safety Profile Assessment of Oncolytic Virus Therapy Based on Clinical Trials. Therapeutic innovation \& regulatory science. $2017 ; 52(4)$

41. Andtbacka RH, Kaufman HL, Collichio F, Amatruda T, Senzer N, Chesney J, et al Talimogene laherparepvec improves durable response rate in patients with advanced melanoma. Journal of clinical Oncology. 2015; 33(25).

42. Irwin CR, Hitt MM, Evans DH. Targeting nucleotide biosynthesis: a strategy for improving the oncolytic potential of DNA viruses. Front Oncol. 2017;7.

43. Delwar ZM, Kuo Y, Wen YH, Rennie, PS, Jia W. Oncolytic virotherapy blockade by microglia and macrophages requires STAT1/3. Cancer research. 2017;78(3)

44. Rye $I H$, Trinh A, Saetersdal A, Nebdal D, Lingjaerde OC, Almendro V, et al. Intra-tumor heterogeneity defines treatment-resistant HER2+ breast tumors. Mol Oncol. 2018

45. Burrell RA, McGranahan N, Bartek J, Swanton C. The causes and consequences of genetic heterogeneity in cancer evolution. Nature. 2013; 501(7467).
46. Sarinella F, Calistri A, Sette P, Palu G, Parolin C. Oncolysis of pancreatic tumour cells by a \%34. 5-deleted HSV-1 does not rely upon Ras-activation, but on the PI 3-kinase pathway. Gene Ther. 2006;13(14).

47. Drost J, Clevers H. Organoids in cancer research. Nat Rev Cancer. 2018;1.

48. Lai Y, Wei X, Lin S, Qin L, Cheng L, Li P. Current status and perspectives of patient-derived xenograft models in cancer research. J Hematol Oncol. 2017;(1):10

49. Carrillo MA, Zhen A, Kitchen SG. The use of the humanized mouse model in gene therapy and immunotherapy for HIV and cancer. Front Immunol. 2018;9.

50. Power AT, Wang J, Falls TJ, Paterson JM, Parato KA, Lichty BD, et al. Carrier cell-based delivery of an oncolytic virus circumvents antiviral immunity. Mol Ther. 2007;15(1)

51. Guo ZS, Liu Z, Bartlett DL. Oncolytic immunotherapy: dying the right way is a key to eliciting potent antitumor immunity. Front Oncol. 2014;4:74.

52. Ricca JM, Oseledchyk A, Walther T, Liu C, Mangarin L, Merghoub T, et al. Pre-existing immunity to oncolytic virus potentiates its immunotherapeutic efficacy. Mol Ther. 2018;26(4).

53. Ribas A, Dummer R, Puzanov I, VanderWalde A, Andtbacka RH, Michielin O, et al. Oncolytic virotherapy promotes intratumoral T cell infiltration and improves anti-PD-1 immunotherapy. Cell. 2017;170(6).

54. Fisher K. Striking out at disseminated metastases: the systemic delivery of oncolytic viruses. Current opinion in molecular therapeutics. 2006; 8(4).

55. Wang Y, Marelli G, Howells A, Lemoine N. Oncolytic viral therapy and the immune system: a double-edged sword against cancer. Front Immunol. 2018;9.

56. Fukuhara $\mathrm{H}$, Ino $\mathrm{Y}$, Todo T. Oncolytic virus therapy: a new era of cancer treatment at dawn. Cancer Sci. 2016:107:10.

57. Liu BL, Robinson M, Han ZQ, Branston RH, English C, Reay P, et al. ICP34.5 deleted herpes simplex virus with enhanced oncolytic, immune stimulating, and anti-tumour properties. Gene Ther. 2003;10(4)

58. Leoni V, Vannini A, Gatta V, Rambaldi J, Sanapo M, Barboni C, et al. A fullyvirulent retargeted oncolytic HSV armed with IL-12 elicits local immunity and vaccine therapy towards distant tumors. PLoS pathogens.2018; 14(8).

59. Kowalsky SJ, Liu Z, Feist M, Berkey SE, Ma C, Ravindranathan R, et al. Superagonist IL-15-Armed Oncolytic Virus Elicits Potent Antitumor Immunity and Therapy That Are Enhanced with PD-1 Blockade. Mol Ther. 2018;(10):26

60. Binnewies M, Roberts EW, Kersten K, Chan V, Fearon DF, Merad M, et al. Understanding the tumor immune microenvironment (TIME) for effective therapy. Nat Med. 2018;1.

61. Hoeller C, Michielin O, Ascierto PA, Szabo Z, Blank CU. Systematic review of the use of granulocyte-macrophage colony-stimulating factor in patients with advanced melanoma. Cancer Immun. 65(9).

62. Kloss CC, Lee J, Zhang A, Chen F, Melenhorst JJ, Lacey SF, et al. Dominant-Negative TGF- $\beta$ Receptor Enhances PSMA-Targeted Human CAR T Cell Proliferation And Augments Prostate Cancer Eradication. Mol Ther. 2018;26(7).

63. Chen CY, Hutzen B, Wedekind MF, Cripe TP. Oncolytic virus and PD-1/PD-L1 blockade combination therapy. Oncolytic virotherapy. 2018;7.

64. Downs-Canner S, Guo ZS, Ravindranathan R, Breitbach CJ, O'malley ME, Jones $\mathrm{HL}$, et al. Phase 1 study of intravenous oncolytic poxvirus (VvDD) in patients with advanced solid cancers. Mol Ther. 2016;24(8).

65. Streby KA, Geller JI, Currier MA, Warren PS, Racadio JM, Towbin AJ, et al. Intratumoral injection of HSV1716, an oncolytic herpes virus, is safe and shows evidence of immune response and viral replication in young cancer patients. Clin Cancer Res. 2017;23(14).

66. Zhang J, Tai LH, Ilkow CS, Alkayyal AA, Ananth AA, De Souza CT, et al. Maraba MG1 virus enhances natural killer cell function via conventional dendritic cells to reduce postoperative metastatic disease. Mol Ther. 2014:22(7).

67. Burke MJ. Oncolytic Seneca Valley Virus: past perspectives and future directions. Oncolytic Virotherapy. 2016;5.

68. Kambara H, Okano H, Chiocca EA, Saeki Y. An oncolytic HSV-1 mutant expressing ICP34.5 under control of a nestin promoter increases survival of animals even when symptomatic from a brain tumor. Cancer Res. 2005; 65(7)

69. Campadelli-Fiume G, Petrovic B, Leoni V, Gianni T, Avitabile E, Casiraghi C, Gatta $V$. Retargeting strategies for oncolytic herpes simplex viruses. Viruses. 2016;8(3) 